\title{
Enhancement of Dissolution rate of Naproxen by Solid Dispersions with cyclodextrin Complex's
}

\author{
Dr.Rohini Pilli ${ }^{1}$, SDVSKiran Kadali ${ }^{2}$, M.V.Nagabhushanam ${ }^{3}$ \\ ${ }^{1}$ AssisstantProfessor, BOS Member in Pharmacy,Department of Pharmaceutics,Acharya Nagarjuna \\ niversity, Guntur,AndhraPradesh,India \\ ${ }^{2}$ Manager,Pharmacovigilance Head,MSN Labs,Hyderbad,Telagana,India ${ }^{3}$ Professor., Hindu College of \\ Pharmacy, Guntur.A.P. India
}

\begin{abstract}
Naproxen is an anti-inflammatory drug that is poorly soluble in water. This paper describes an approach to improve the dissolution rate of Naproxen by using solid dispersions (SDs) in hydrophilic polymers The solid dispersions prepared with a Co-evaporation, kneaded method \& Physical Mixture method using different concentrations of $\alpha$-cyclodextrin $(\alpha-C D)$. The release of Naproxen from various solid dispersions was determined from dissolution studies by use of USP dissolution apparatus.The dissolution study results revealed that there was a considerable increase in solubility of all solid dispersions as compared to pure drug. Prepared Solid dispersions were characterized by DSC, PXRD, IR and SEM images were evaluated for drug content, saturation solubility. Physicochemical characterization of solid dispersions suggests a reduction in drug crystallinity following dissolution enhancement. Results indicate that present \%DE 30 of drug was improved from 36.34 to75.04 by the use of Naproxen $\alpha$-CD-HPMC (1:2:0.3) Kneaded complex.
\end{abstract}

Keywords:Naproxen Solid dispersions, hydrophilic polymers

\section{INTRODUCTION}

Naproxen is a Proprionic acid derivative related to the Arylacetic acid group of NonSteroidal AntiInflammatory drugs ${ }^{[1]}$..The chemical names for Naproxen and Naproxen Sodium are (S)-6-Methoxy-A-Methyl2-Naphthaleneacetic Acid and (S)-6-Methoxy-a-Methyl-2-Naphthaleneacetic Acid, Sodium salt, respectively ${ }^{[1,2]}$.. Naproxen and Naproxen Sodium have the following structures, respectively<smiles>[2H]C([2H])(C)c1ccc2cc(OC)ccc2c1</smiles>

\section{PREPARATION OF SOLID DISPERSIONS}

In each case solid complexes of drug and cyclodextrin were prepared in 1:1, 1:1:0.2, 1:2 \&1:2:0.3 ratios by three methods kneading, co evaporation and physical mixture.

\section{Kneading Method}

Drug and cyclodextrin with or without auxiliary substances (PEG, PVP, HPMC) were triturated in a mortar with a small volume of water. After wetting the mixture in a mortar, the thick slurry was kneaded for 45 minutes and then dried at $55^{\circ} \mathrm{C}$ until dry. The dried mass was pulverized, sieved through sieve no.120 and stored in desiccators till further use.

\section{Coevaporation Method}

Drug with or without auxiliary substances (PEG, PVP, HPMC) were dissolved in methanol, stirred the solution. The solvent was removed at reduced pressure in rotary evaporator at $45^{\circ} \mathrm{C}$ for 3 hours and dried mass was pulverized, sieved through sieve no.120 and stored in desiccators till further use $\mathrm{e}^{[3,4]}$.

\section{Physical Mixture}

The Physical mixtures were prepared by gently mixing drug, cyclodextrin with or without auxiliary substance (PEG, PVP and HPMC), in a mortar with pestle for 10 minutes. These mixtures were passed through a sieve no.120 and stored in desiccators till further use. 
TABLE.1: Composition of various Solid Dispersions Prepared

\begin{tabular}{|c|c|c|c|}
\hline \multirow{2}{*}{$\begin{array}{l}\text { Sl. } \\
\text { No. }\end{array}$} & \multicolumn{3}{|c|}{ Composition } \\
\hline & Drug & Carriers & SD Code \\
\hline 1. & Naproxen & $\alpha-\mathrm{CD}$ & N- $\alpha-C D(1: 1)$ \\
\hline 2. & Naproxen & $\alpha-\mathrm{CD}, \mathrm{PEG}$ & $\mathrm{N}-\alpha-\mathrm{CD}, \mathrm{PEG}(1: 1: 0.2)$ \\
\hline 3. & Naproxen & $\alpha-\mathrm{CD}, \mathrm{PVP}$ & $\mathrm{N}-\alpha-\mathrm{CD}, \mathrm{PVP}(1: 1: 0.2)$ \\
\hline 4. & Naproxen & $\alpha-\mathrm{CD}, \mathrm{HPMC}$ & N- $\alpha$-CD ,HPMC (1:1:0.2) \\
\hline 5 & Naproxen & $\alpha-\mathrm{CD}$ & N- $\alpha-C D(1: 2)$ \\
\hline 6 & Naproxen & $\alpha-\mathrm{CD}, \mathrm{PEG}$ & $\mathrm{N}-\alpha-\mathrm{CD}, \mathrm{PEG}(1: 2: 0.3)$ \\
\hline 7 & Naproxen & $\alpha-C D, P V P$ & N- $\alpha$-CD ,PVP (1:2:0.3) \\
\hline 8 & Naproxen & $\alpha-\mathrm{CD}, \mathrm{HPMC}$ & $\mathrm{N}-\alpha-\mathrm{CD}, \mathrm{HPMC}(1: 2: 0.3)$ \\
\hline
\end{tabular}

ESTIMATION OF NAPROXEN IN SOLID DISPERSIONS

$100 \mathrm{mg}$ of inclusion complex was taken in a $50 \mathrm{ml}$ volumetric flask. Methanol about $40 \mathrm{ml}$ was added and mixed thoroughly. The contents were repeatedly warmed in a hot bath while mixing to dissolve the drug in the solvent $^{[6,7]}$. The solution was made up to volume with methanol. The solution was then suitably diluted and assayed for drug content by the specific spectrophotometric method

TABLE 2: Drug Content of Solid Inclusion Complexes of Naproxen $\square$-CD, Prepared by Kneading ,Coevaporation and Physical Mixture Methods

\begin{tabular}{|c|c|c|c|}
\hline \multirow[b]{2}{*}{ CD Complex } & \multicolumn{3}{|c|}{ Percent Naproxen Content ( $\bar{x} \pm$ s.d., $)$} \\
\hline & $\begin{array}{l}\text { Kneading } \\
\text { Method }\end{array}$ & $\begin{array}{l}\text { Coevaporation } \\
\text { Method }\end{array}$ & PhysicalMixture \\
\hline $\mathrm{N}-\alpha \mathrm{CD}(1: 1)$ & $49.89 \pm 0.71$ & $49.79 \pm 0.86$ & $49.95 \pm 0.82$ \\
\hline N- $\alpha$ CD:PEG (1:1:0.2) & $45.84 \pm 0.94$ & $45.98 \pm 0.88$ & $45.40 \pm 0.89$ \\
\hline N- $\alpha$ CD:PVP (1:1:0.2) & $45.78 \pm 0.77$ & $44.60 \pm 0.78$ & $45.88 \pm 0.54$ \\
\hline N- $\alpha$ CD:HPMC (1:1:0.2) & $45.37 \pm 0.84$ & $45.56 \pm 0.92$ & 44.93 \pm 0.94 \\
\hline $\mathrm{N}-\alpha \mathrm{CD}(1: 2)$ & $33.34 \pm 0.78$ & $33.38 \pm 0.65$ & $33.30 \pm 0.85$ \\
\hline $\mathrm{N}-\alpha$ CD:PEG (1:2:0.3) & $30.59 \pm 0.69$ & 30.79 \pm 0.89 & $29.70 \pm 0.77$ \\
\hline N- $\alpha$ CD:PVP (1:2:0.3) & $30.63 \pm 0.93$ & $30.27 \pm 0.73$ & $30.39 \pm 0.95$ \\
\hline N- $\alpha$ CD:HPMC (1:2:0.3) & $30.27 \pm 0.88$ & $30.35 \pm 0.78$ & $30.29 \pm 0.38$ \\
\hline
\end{tabular}

\section{X-Ray Powder Diffractometry (Xrd)}

The X-Ray diffractograms of pure drug naproxen exhibited characteristic diffraction pattern indicating their crystalline nature. X-ray diffractograms of the pure drugs and their cyclodextrin complexes are shown in Fig.

21

\section{Differential scanning calorimetry}

The DSC curve of naproxen showed a single sharp exothermic peak at $158.9^{0} \mathrm{C}$ corresponding to its melting point. $\alpha \mathrm{CD}, \mathrm{HPMC}$ showed a broad peaks associated with loss of water. In the DSC thermograms of naproxen $\alpha$-CD-HPMC the intensity or height of the exothermic peaks at $153.7^{\circ} \mathrm{C}$ respectively were reduced indicating interaction of naproxen with cyclodextrins. The change in symmetry of the peak clearly indicates the formation of a complex. The exothermic peak of the cyclodextrin complexes of naproxen at $153.7^{0} \mathrm{C}$ was markedly reduced indicating the reduction of crystalline nature of drug and its complexation and amorphization with cyclodextrins are shown im Fig.20

\section{Fourier-transform infrared spectroscopy (FTIR)}

The principal IR absorption peaks of naproxen at $2968 \mathrm{~cm}^{-1}$ (Ar-C-H stretch), $2829 \mathrm{~cm}^{-1}(\mathrm{COOH}$ stretch), $3543 \mathrm{~cm}^{-1}$ (OH stretch in $\left.\mathrm{COOH}\right), 3092 \mathrm{~cm}^{-1}\left(\mathrm{CH}\right.$ stretch in $\left.\mathrm{CH}_{3}\right), 1157 \mathrm{~cm}^{-1}$ (C-O-C stretching), 1717 $\mathrm{cm}^{-1}(\mathrm{C}=\mathrm{O}$ stretch in $\mathrm{COOH}), 1387 \mathrm{~cm}^{-1}(\mathrm{CH}$ bending in $\mathrm{Ar}-\mathrm{CH}), 1596 \mathrm{~cm}^{-1}(\mathrm{C}=\mathrm{C}$ stretching $)$ were all observed in the spectra of naproxen and its cyclodextrin complex N-HP $\beta$-CD-HPMC. IR absorption peaks of naproxen , HPMC and its cyclodextrin complexes are shown in Fig.19 


\section{Scanning electron microscopy (SEM) studies}

The surface morphology was examined by Scanning electron microscopic studies and the photographs are shown in Fig. 22. SEM is used to study the microscopic aspects of the raw materials like pure drug, $\alpha-C D$ and the complexation products obtained from different methods of preparation. The pictures were then taken at an excitation voltage of $15 \mathrm{kV}$. SEM images of naproxen and its cyclodextrin complexes ${ }^{[8,9]}$.

\section{Dissolution Rate Studies on Solid Dispersions:}

Dissolution rate of drug from the CD complexes was studied using USP XXIII -6 station dissolution rate test apparatus (ElectroLab) with a paddle stirrer at specified rpm and a temperature of $37^{0} \pm 1^{0} \mathrm{C}$ with $0.1 \mathrm{~N}$ $\mathrm{HCl}$, ( $\mathrm{pH}$ 1.2) Drug or drug-CD complex equivalent to $100 \mathrm{mg}$ of naproxen was used in each dissolution rate test.

Samples of dissolution medium $(5 \mathrm{ml})$ were withdrawn through a filter $(0.45 \mu)$ at different time intervals, suitably diluted and assayed for the drug content by measuring absorbance at $272 \mathrm{~nm}$ in the case of naproxe ${ }^{[10,11,12]}$.. The dissolution fluid withdrawn at each sampling time was replaced with fresh dissolution fluid in each case. Dissolution experiments were conducted in triplicate in each case.

\section{Dissolution Profiles of Naproxen and its Solid Dispersions}

\section{TABLE.3: Dissolution Profiles of Naproxen and its $\square$-CD Complexes Prepared by Kneading Method}

\begin{tabular}{|c|c|c|c|c|c|}
\hline & \multicolumn{6}{|c|}{ Percent Naproxen Dissolved( $x$ s.d., $\mathrm{n}=3)$} \\
\cline { 2 - 6 } & $\mathrm{N}$ & $\mathrm{N}: \alpha \mathrm{CD} 1: 1$ & $\begin{array}{c}\mathrm{N}: \alpha \mathrm{CD}: \mathrm{PEG} \\
1: 1: 0.2\end{array}$ & $\begin{array}{c}\mathrm{N}: \alpha \mathrm{CD}: \mathrm{PVP} \\
1: 1: 0.2\end{array}$ & $\begin{array}{l}\mathrm{N}: \alpha \mathrm{CD}: \mathrm{HPMC} \\
1: 1: 0.2\end{array}$ \\
\hline 0 & 0 & 0 & 0 & 0 & 0 \\
\hline 5 & $20.13 \pm 0.92$ & $30.32 \pm 0.98$ & $34.56 \pm 0.90$ & $40.66 \pm 0.90$ & $50.32 \pm 0.90$ \\
\hline 10 & $23.14 \pm 0.95$ & $40.63 \pm 0.95$ & $42.13 \pm 0.91$ & $45.89 \pm 0.91$ & $56.83 \pm 0.91$ \\
\hline 20 & $25.32 \pm 0.93$ & $41.83 \pm 0.91$ & $45.43 \pm 0.94$ & $50.36 \pm 0.94$ & $61.42 \pm 0.94$ \\
\hline 30 & $26.12 \pm 0.96$ & $43.16 \pm 0.94$ & $46.32 \pm 0.98$ & $55.89 \pm 0.97$ & $66.34 \pm 0.98$ \\
\hline 45 & $28.7 \pm 0.94$ & $48.32 \pm 0.92$ & $51.35 \pm 0.97$ & $63.16 \pm 0.98$ & $69.53 \pm 0.97$ \\
\hline 60 & $29.97 \pm 0.91$ & $51.64 \pm 0.93$ & $57.46 \pm 0.94$ & $68.49 \pm 0.95$ & $75.13 \pm 0.94$ \\
\hline 90 & $34.45 \pm 0.93$ & $54.36 \pm 0.90$ & $61.66 \pm 0.95$ & $70.56 \pm 0.96$ & $80.93 \pm 0.92$ \\
\hline 120 & $43.05 \pm 0.98$ & $56.83 \pm 0.91$ & $66.85 \pm 0.91$ & $77.32 \pm 0.93$ & $84.16 \pm 0.93$ \\
\hline
\end{tabular}

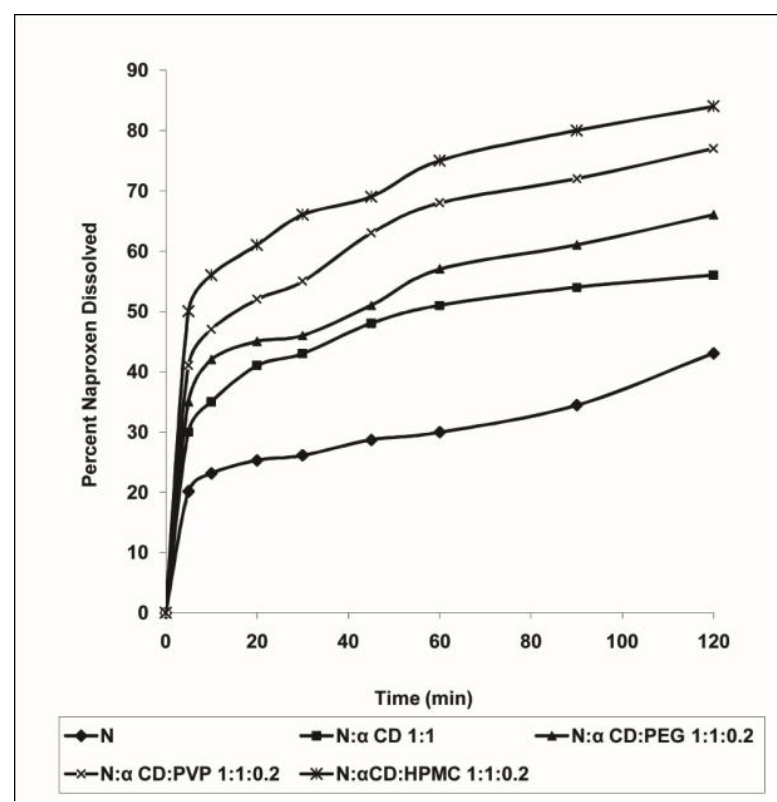

Fig. 1.Dissolution Profiles of Naproxen and its $\square$-CD Complexes Prepared by Kneading Method 


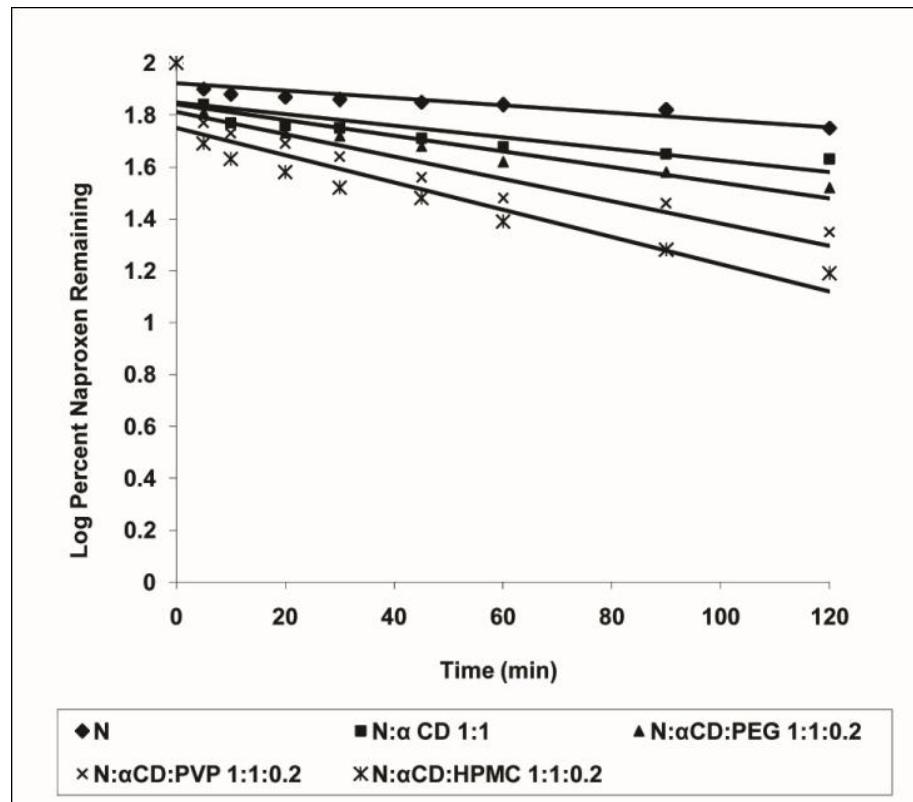

Fig. 2.First Order Dissolution Plots of Naproxen and its $\square$-CD Complexes Prepared by Kneading Method

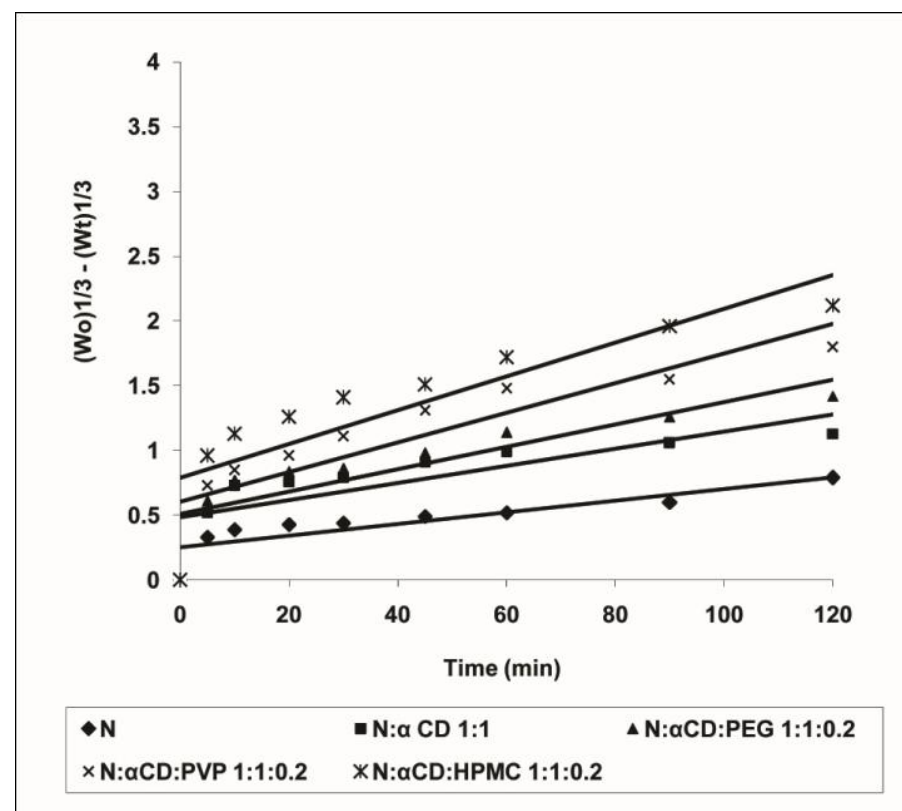

Fig. 3. Hixson Crowell Plots of Naproxen and its $\square$-CD complexes Prepared by Kneading Method

Table.4. Dissolution Profiles of Naproxen and its $\square$ - CD prepared by Kneading Method

\begin{tabular}{|c|c|c|c|c|c|}
\hline \multirow{2}{*}{$\begin{array}{c}\text { TIME } \\
(\text { min) }\end{array}$} & \multicolumn{5}{|c|}{ Percent Naproxen Dissolved( $\overline{\boldsymbol{x}} \pm$ s.d., n=3) } \\
\cline { 2 - 6 } & $\mathbf{N}$ & $\begin{array}{c}\text { N: } \square \text { CD } \\
\mathbf{1 : 2}\end{array}$ & $\begin{array}{c}\text { N: } \\
\text { CD:PEG } \\
\mathbf{1 : 2 : 0 . 3}\end{array}$ & $\begin{array}{c}\text { N: } \square \text { CD:PVP } \\
\mathbf{1 : 2 : 0 . 3}\end{array}$ & $\begin{array}{l}\text { N: } \square \text { CD:HPMC } \\
\mathbf{1 : 2 : 0 . 3}\end{array}$ \\
\hline 0 & 0 & 0 & 0 & 0 & 0 \\
\hline 5 & $20.13 \pm 0.92$ & $53.16 \pm 0.89$ & $58.32 \pm 0.89$ & $64.16 \pm 0.96$ & $70.32 \pm 0.68$ \\
\hline 10 & $23.14 \pm 0.95$ & $62.53 \pm 0.95$ & $66.31 \pm 0.96$ & $73.56 \pm 0.94$ & $79.16 \pm 0.87$ \\
\hline 20 & $25.32 \pm 0.93$ & $66.13 \pm 0.97$ & $74.16 \pm 0.75$ & $80.62 \pm 0.92$ & $86.63 \pm 0.86$ \\
\hline 30 & $26.12 \pm 0.96$ & $73.54 \pm 0.93$ & $85.33 \pm 0.82$ & $88.68 \pm 0.87$ & $92.96 \pm 0.98$ \\
\hline 45 & $28.7 \pm 0.94$ & $80.16 \pm 0.95$ & $90.32 \pm 0.86$ & $92.16 \pm 0.78$ & $96.98 \pm 0.91$ \\
\hline
\end{tabular}




\begin{tabular}{|c|c|c|c|c|c|}
\hline 60 & $29.97 \pm 0.91$ & $83.54 \pm 0.93$ & $92.13 \pm 0.88$ & $95.32 \pm 0.76$ & $98.33 \pm 0.90$ \\
\hline 90 & $34.45 \pm 0.93$ & $85.61 \pm 0.98$ & $94.16 \pm 0.87$ & $96.13 \pm 0.98$ & $99.44 \pm 0.88$ \\
\hline 120 & $43.05 \pm 0.98$ & $88.23 \pm 0.92$ & $95.32 \pm 0.80$ & $98.15 \pm 0.88$ & $99.86 \pm 0.97$ \\
\hline
\end{tabular}

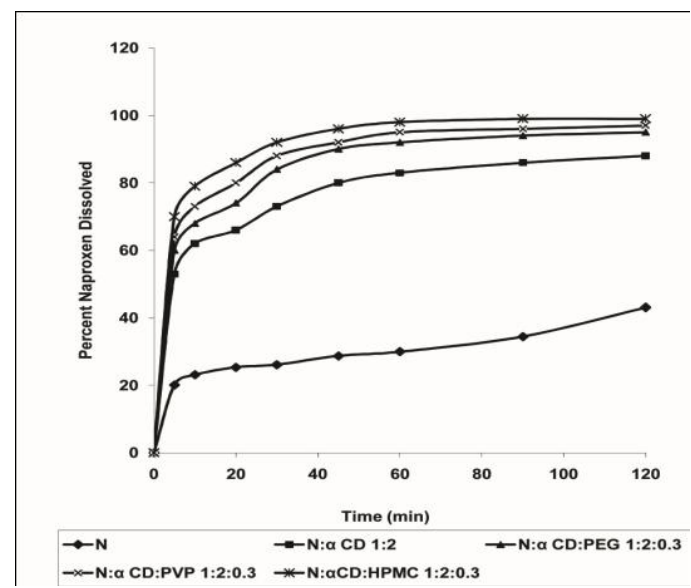

Fig .4 Dissolution Profiles of Naproxen and its $\square$ - CD complexes prepared by Kneading Method

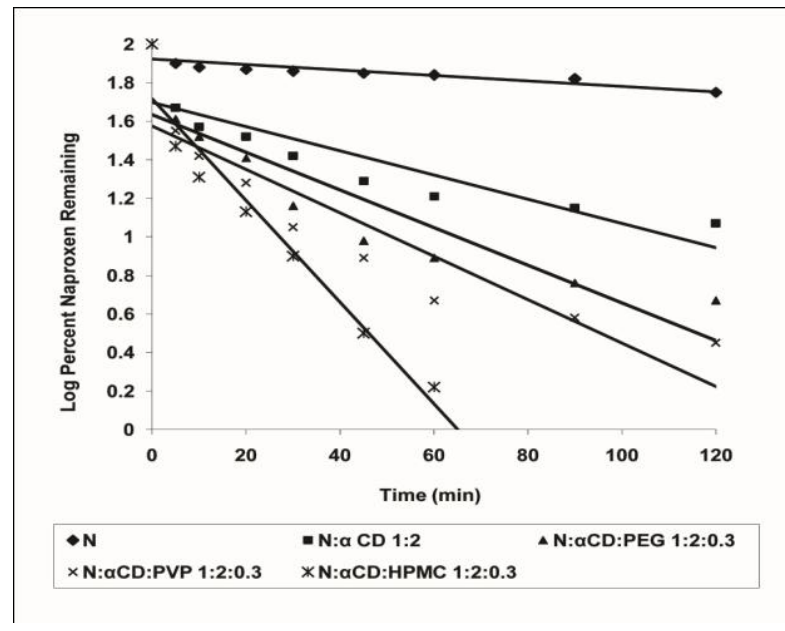

Fig. 5. First Order Dissolution Plots of Naproxen and its $\square$ - CD Complexes prepared by Kneading Method

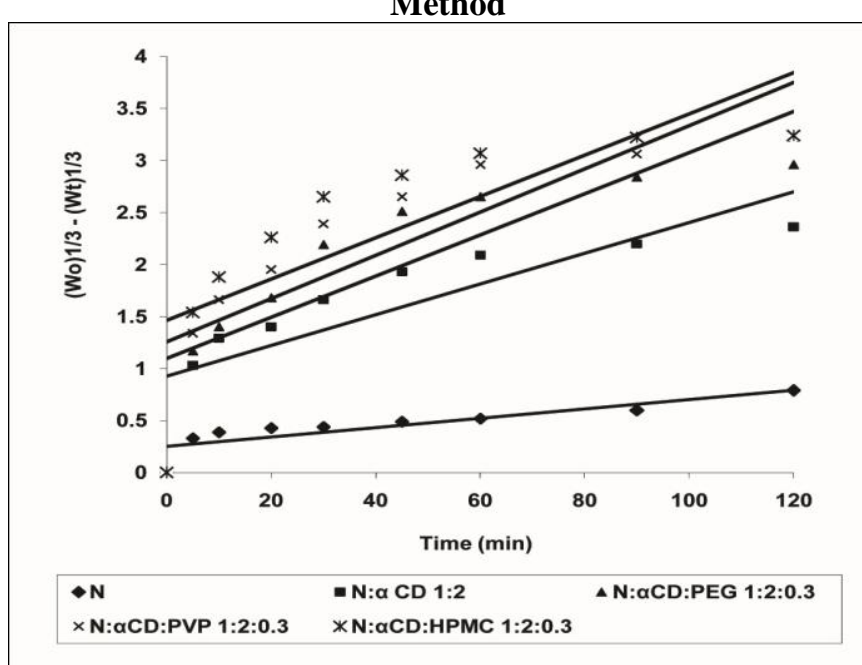

Fig.6. Hixson Crowel Dissolution Plots of Naproxen and its $\square$ - CD Complexes prepared by Kneading Method 
TABLE 5. Dissolution Profiles of Naproxen and its $\square$-C D Complexes Prepared by Co Evaporation Method

\begin{tabular}{|c|c|c|c|c|c|}
\hline \multirow{2}{*}{$\begin{array}{l}\text { TIME } \\
\text { (min) }\end{array}$} & \multicolumn{5}{|c|}{ Percent Naproxen Dissolved ( $\bar{x} \pm$ s.d., $n=3$ ) } \\
\hline & $\mathbf{N}$ & N: $\square$ CD 1:1 & $\begin{array}{c}\text { N: } \square \text { CD:PEG } \\
\text { 1:1:0.2 } \\
\end{array}$ & $\begin{array}{c}\mathrm{N}: \square \text { CD:PVP } \\
\text { 1:1:0.2 } \\
\end{array}$ & $\begin{array}{c}\mathrm{N}: \square \text { CD:HPMC } \\
1: 1: 0.2 \\
\end{array}$ \\
\hline 0 & 0 & 0 & 0 & 0 & 0 \\
\hline 5 & $20.13 \pm 0.92$ & $26.23 \pm 0.92$ & $30.65 \pm 0.960$ & $33.65 \pm 0.91$ & $37.23 \pm 0.93$ \\
\hline 10 & $23.14 \pm 0.95$ & $37.36 \pm 0.93$ & $39.31 \pm 0.91$ & $42.98 \pm 0.92$ & $45.38 \pm 0.94$ \\
\hline 20 & $25.32 \pm 0.93$ & $39.38 \pm 0.95$ & $41.34 \pm 0.94$ & $47.63 \pm 0.94$ & $52.34 \pm 0.92$ \\
\hline 30 & $26.12 \pm 0.96$ & $40.61 \pm 0.94$ & $42.23 \pm 0.95$ & $49.98 \pm 0.98$ & $56.43 \pm 0.91$ \\
\hline 45 & $28.7 \pm 0.94$ & $44.33 \pm 0.98$ & $47.53 \pm 0.92$ & $53.61 \pm 0.96$ & $59.35 \pm 0.98$ \\
\hline 60 & $29.97 \pm 0.91$ & $49.46 \pm 0.97$ & $51.64 \pm 0.98$ & $58.94 \pm 0.94$ & $65.31 \pm 0.97$ \\
\hline 90 & $34.45 \pm 0.93$ & $51.63 \pm 0.91$ & $57.66 \pm 0.97$ & $60.65 \pm 0.95$ & $70.39 \pm 0.96$ \\
\hline 120 & $43.05 \pm 0.98$ & $54.39 \pm 0.92$ & $59.58 \pm 0.93$ & $65.23 \pm 0.91$ & $73.61 \pm 0.91$ \\
\hline
\end{tabular}

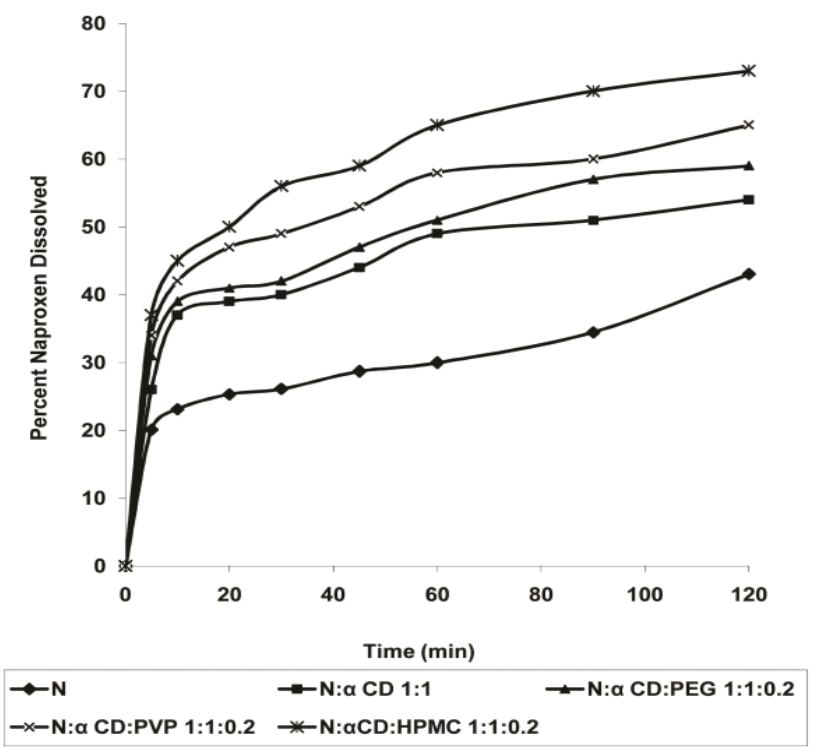

Fig .5.129Dissolution Profiles of Naproxen and its $\alpha$ - Cyclodextrin complexes prepared by Coevaporation Method

Fig.7.DissolutionProfiles of Naproxen and its $\square$-CD Complexes Prepared by Coevaporation Method

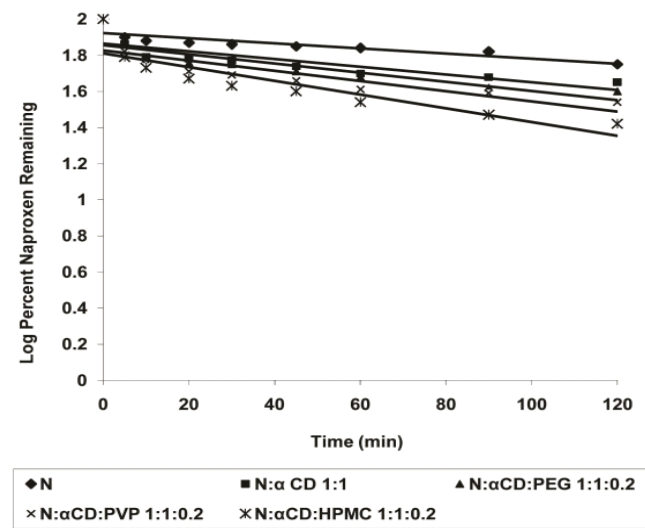


Fig.8. First Order Dissolution Plots of Naproxen and its $\square$-CD Complexes Prepared by Coevaporation Method

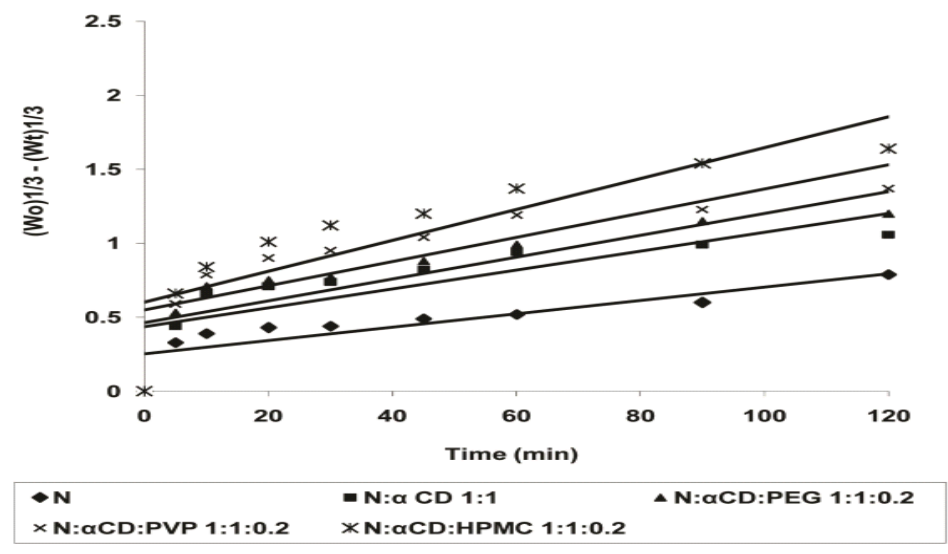

Fig.5.131 Hixson Crowel Dissolution Plots of Naproxen and its Cyclodextrin complexe prepared by Coevaporation Method

Fig.9. Hixson Crowell Plots of Naproxen and its $\square$-CD complexes Prepared by Coevaporation Method TABLE.6. Dissolution Profiles of Naproxen and its $\square$-CD Complexes Prepared by Co Evaporation Method

\begin{tabular}{|c|c|c|c|c|c|}
\hline \multirow{2}{*}{$\begin{array}{c}\text { TIME } \\
(\text { min) }\end{array}$} & \multicolumn{5}{|c|}{ Percent Naproxen Dissolved ( $\bar{x} \pm$ s.d.,n =3) } \\
\cline { 2 - 6 } & $\mathbf{N}$ & N: $\square$ CD 1:2 & $\begin{array}{c}\text { N: } \square \\
\text { CD:PEG } \\
\mathbf{1 : 2 : 0 . 3}\end{array}$ & $\begin{array}{c}\text { N: } \square \text { CD:PVP } \\
\mathbf{1 : 2 : 0 . 3}\end{array}$ & $\begin{array}{c}\text { N: } \square \text { CD:HPMC } \\
\mathbf{1 : 2 : 0 . 3}\end{array}$ \\
\hline 0 & 0 & 0 & 0 & 0 & 0 \\
\hline 5 & $20.13 \pm 0.92$ & $44.61 \pm 0.98$ & $49.32 \pm 0.98$ & $53.61 \pm 0.91$ & $58.23 \pm 0.90$ \\
\hline 10 & $23.14 \pm 0.95$ & $52.35 \pm 0.96$ & $56.31 \pm 0.96$ & $62.65 \pm 0.97$ & $66.61 \pm 0.94$ \\
\hline 20 & $25.32 \pm 0.93$ & $56.31 \pm 0.92$ & $63.61 \pm 0.94$ & $68.26 \pm 0.90$ & $70.36 \pm 0.96$ \\
\hline 30 & $26.12 \pm 0.96$ & $62.94 \pm 0.94$ & $73.32 \pm 0.90$ & $75.86 \pm 0.95$ & $77.69 \pm 0.93$ \\
\hline 45 & $28.7 \pm 0.94$ & $70.61 \pm 0.91$ & $80.23 \pm 0.95$ & $84.61 \pm 0.96$ & $80.89 \pm 0.92$ \\
\hline 60 & $29.97 \pm 0.91$ & $73.45 \pm 0.90$ & $82.31 \pm 0.93$ & $85.13 \pm 0.93$ & $85.32 \pm 0.94$ \\
\hline 90 & $34.45 \pm 0.93$ & $75.16 \pm 0.92$ & $84.61 \pm 0.97$ & $87.31 \pm 0.94$ & $86.74 \pm 0.95$ \\
\hline 120 & $43.05 \pm 0.98$ & $78.32 \pm 0.97$ & $85.23 \pm 0.91$ & $88.57 \pm 0.93$ & $86.68 \pm 0.90$ \\
\hline
\end{tabular}

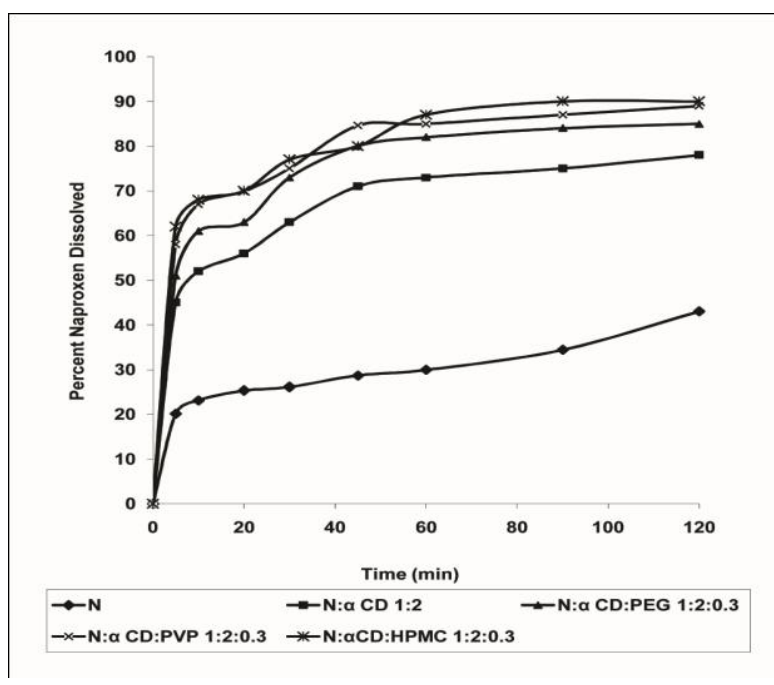


Fig.10. Dissolution Profiles of Naproxen and its $\square$-CD Complexes Prepared by Coevaporation Method

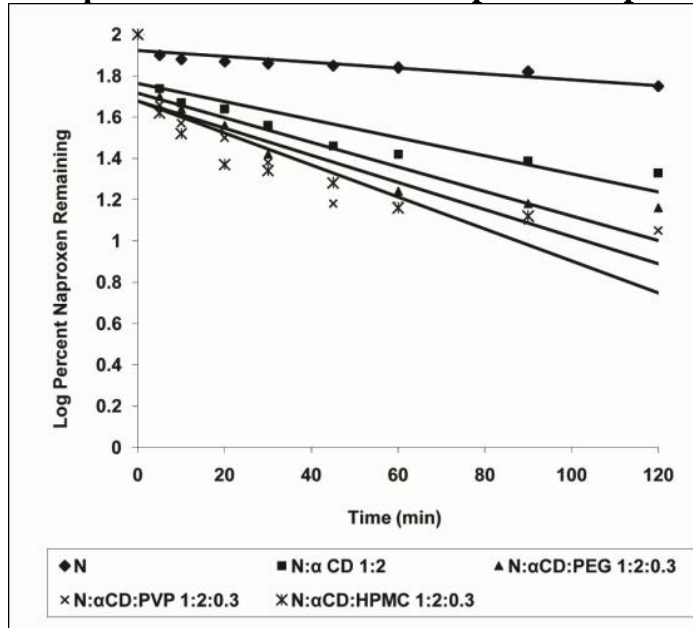

Fig. 11.First Order Dissolution Plots of Naproxen and its $\square$-CD Complexes Prepared by Co Evaporation Method

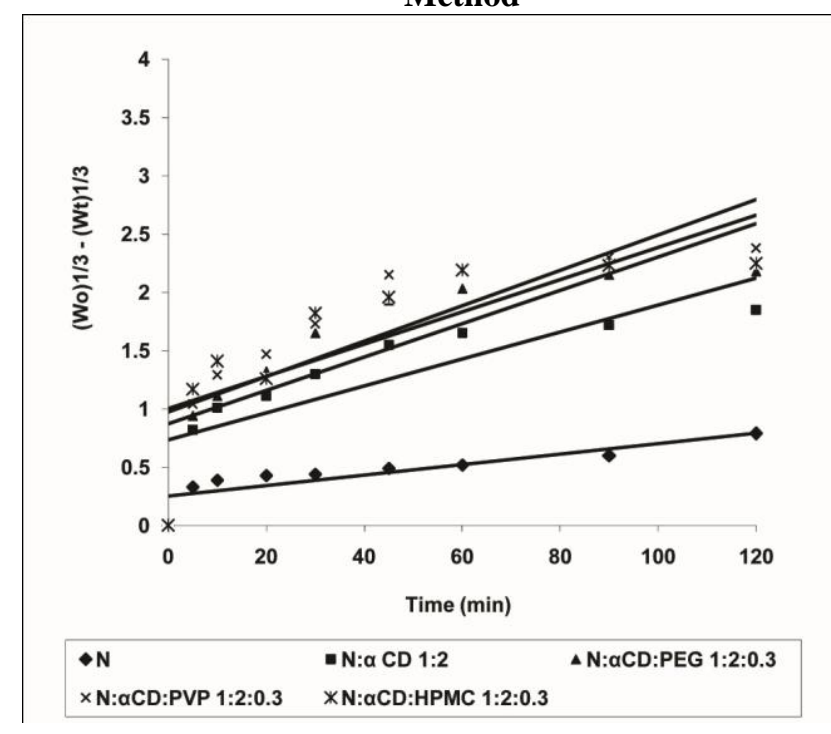

.Fig.12.Hixson Crowell Plots of Naproxen and its $\square$-CD complexes Prepared by CoevaporationMethod

TABLE :7.Dissolution Profiles of Naproxen and its $\square$-CD Complexes Prepared by Physical Mixture Method

\begin{tabular}{|c|c|c|c|c|c|}
\hline \multirow{2}{*}{$\begin{array}{l}\text { TIME } \\
(\mathbf{m i n})\end{array}$} & \multicolumn{5}{|c|}{ Percent Naproxen Dissolved ( $\bar{x} \pm$ s.d., $n=3$ ) } \\
\hline & $\mathbf{N}$ & $\begin{array}{c}\mathrm{N}: \square \mathrm{CD} \\
1: 1\end{array}$ & $\begin{array}{c}\text { N: } \\
\square \text { CD:PEG } \\
\text { 1:1:0.2 }\end{array}$ & $\begin{array}{c}\mathrm{N}: \square \text { CD:PVP } \\
1: 1: 0.2\end{array}$ & $\begin{array}{c}\mathrm{N}: \square \text { CD:HPMC } \\
1: 1: 0.2\end{array}$ \\
\hline 0 & 0 & 0 & 0 & 0 & 0 \\
\hline 5 & $20.13 \pm 0.92$ & $22.56 \pm 0.98$ & $24.86 \pm 0.96$ & $25.56 \pm 0.92$ & $28.32 \pm 0.97$ \\
\hline 10 & $23.14 \pm 0.95$ & $30.63 \pm 0.89$ & $33.13 \pm 0.97$ & $36.88 \pm 0.93$ & $40.83 \pm 0.95$ \\
\hline 20 & $25.32 \pm 0.93$ & $32.83 \pm 0.92$ & $35.43 \pm 0.90$ & $39.33 \pm 0.92$ & $42.43 \pm 0.94$ \\
\hline 30 & $26.12 \pm 0.96$ & $35.16 \pm 0.91$ & $38.61 \pm 0.91$ & $40.36 \pm 0.91$ & $44.34 \pm 0.95$ \\
\hline 45 & $28.7 \pm 0.94$ & $37.33 \pm 0.95$ & $41.35 \pm 0.92$ & $44.16 \pm 0.93$ & $48.53 \pm 0.96$ \\
\hline 60 & $29.97 \pm 0.91$ & $39.64 \pm 0.92$ & $44.46 \pm 0.91$ & $45.49 \pm 0.94$ & $51.13 \pm 0.95$ \\
\hline 90 & $34.45 \pm 0.93$ & $42.23 \pm 0.90$ & $46.65 \pm 0.90$ & $49.93 \pm 0.96$ & $55.93 \pm 0.97$ \\
\hline 120 & $43.05 \pm 0.98$ & $44.83 \pm 0.89$ & $48.85 \pm 0.90$ & $51.23 \pm 0.97$ & $58.16 \pm 0.98$ \\
\hline
\end{tabular}




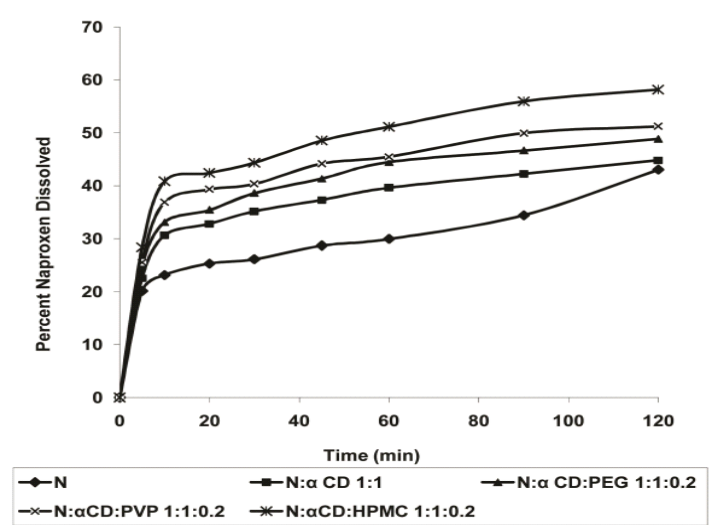

Fig .5.159Dissolution Profiles of Naproxen and it's $\alpha$-Cyclodextrin complexes prepared by

Fig. 13.Dissolution Profiles of Naproxen and its $\square$-CD Complexes Prepared by Physical mixture Method

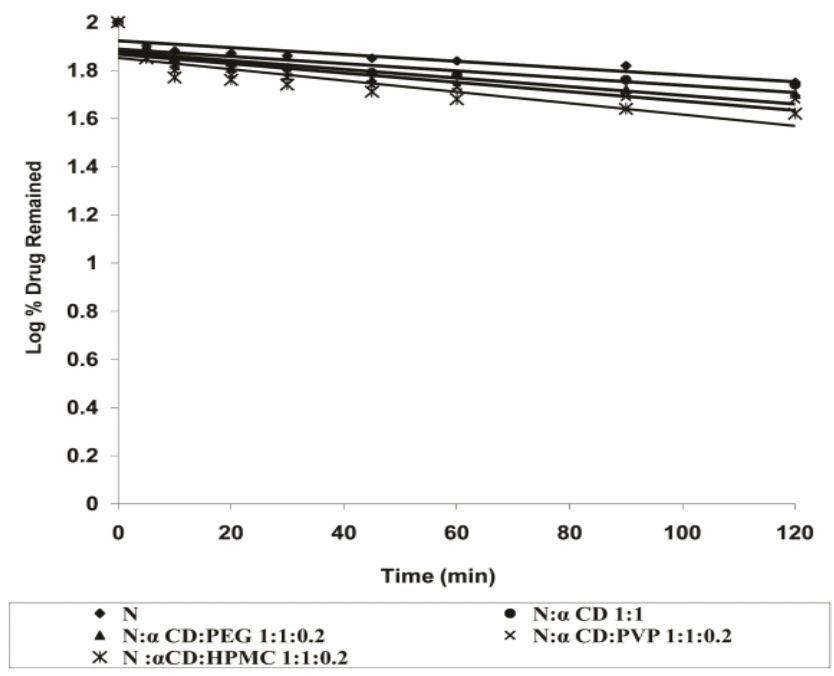

Fig .5.160 First Order Dissolution Plots of Naproxen and it's $\alpha$ - Cyclodextrin complexes

prepared by Physical Mixing Method

Fig. 14.First Order Dissolution Plots of Naproxen and its $\square$-CD Complexes Prepared by Physical mixture Method

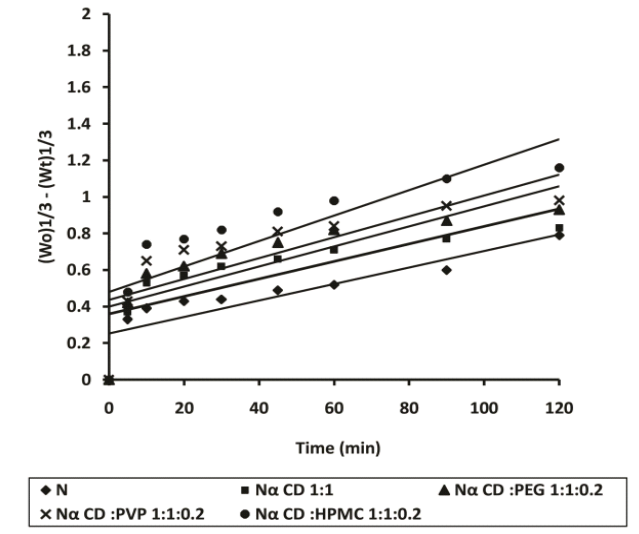

Fig .5.161 Hixson-Crowell Plots of Naproxen and it's $\alpha$ - Cyclodextrin complexes prepared

Fig. 15. Hixson Crowell Plots of Naproxen and its $\square$-CD complexes Prepared by Physical mixture Method 
TABLE 8:Dissolution Profiles of Naproxen and its $\square$-CD Complexes Prepared by Physical Mixture Method

\begin{tabular}{|c|c|c|c|c|c|}
\hline \multirow{2}{*}{$\begin{array}{l}\text { TIME } \\
\text { (min) }\end{array}$} & \multicolumn{5}{|c|}{ Percent Naproxen Dissolved ( $\bar{x} \pm$ s.d., $n=3$ ) } \\
\hline & $\mathbf{N}$ & $\begin{array}{c}\mathrm{N}: \square \mathrm{CD} \\
1: 2\end{array}$ & $\begin{array}{c}\text { N: } \\
\text { CD:PEG } \\
\text { 1:2:0.3 }\end{array}$ & $\begin{array}{c}\mathrm{N}: \square \text { CD:PVP } \\
1: 2: 0.3\end{array}$ & $\begin{array}{c}\mathrm{N}: \square \text { CD:HPMC } \\
1: 2: 0.3\end{array}$ \\
\hline 0 & 0 & 0 & 0 & 0 & 0 \\
\hline 5 & $20.13 \pm 0.92$ & $30.16 \pm 0.93$ & $35.66 \pm 0.95$ & $38.16 \pm 0.94$ & $43.32 \pm 0.94$ \\
\hline 10 & $23.14 \pm 0.95$ & $38.38 \pm 0.90$ & $40.93 \pm 0.92$ & $42.56 \pm 0.98$ & $48.38 \pm 0.92$ \\
\hline 20 & $25.32 \pm 0.93$ & $42.13 \pm 0.89$ & $45.16 \pm 0.90$ & $45.62 \pm 0.96$ & $51.34 \pm 0.91$ \\
\hline 30 & $26.12 \pm 0.96$ & $44.49 \pm 0.91$ & $53.94 \pm 0.91$ & $55.68 \pm 0.92$ & $58.96 \pm 0.91$ \\
\hline 45 & $28.7 \pm 0.94$ & $47.16 \pm 0.83$ & $58.32 \pm 0.95$ & $58.23 \pm 0.95$ & $61.89 \pm 0.90$ \\
\hline 60 & $29.97 \pm 0.91$ & $53.54 \pm 0.92$ & $60.45 \pm 0.92$ & $62.91 \pm 0.90$ & $66.97 \pm 0.89$ \\
\hline 90 & $34.45 \pm 0.93$ & $56.61 \pm 0.97$ & $64.16 \pm 0.93$ & $64.16 \pm 0.96$ & $69.44 \pm 0.88$ \\
\hline 120 & $43.05 \pm 0.98$ & $60.32 \pm 0.93$ & $66.23 \pm 0.95$ & $68.75 \pm 0.92$ & $73.86 \pm 0.89$ \\
\hline
\end{tabular}

Fig.16.DissolutionProfiles of Naproxen and its $\square$-CD Complexes Prepared by Physical Mixture Method

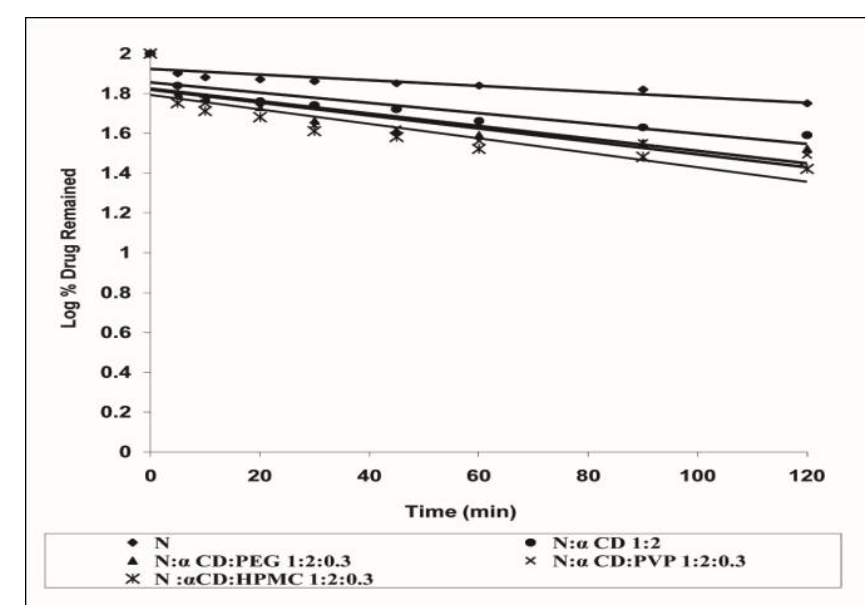

Fig.17. First Order Dissolution Plots of Naproxen and its $\square$-CD Complexes Prepared by Physical Mixture Method 


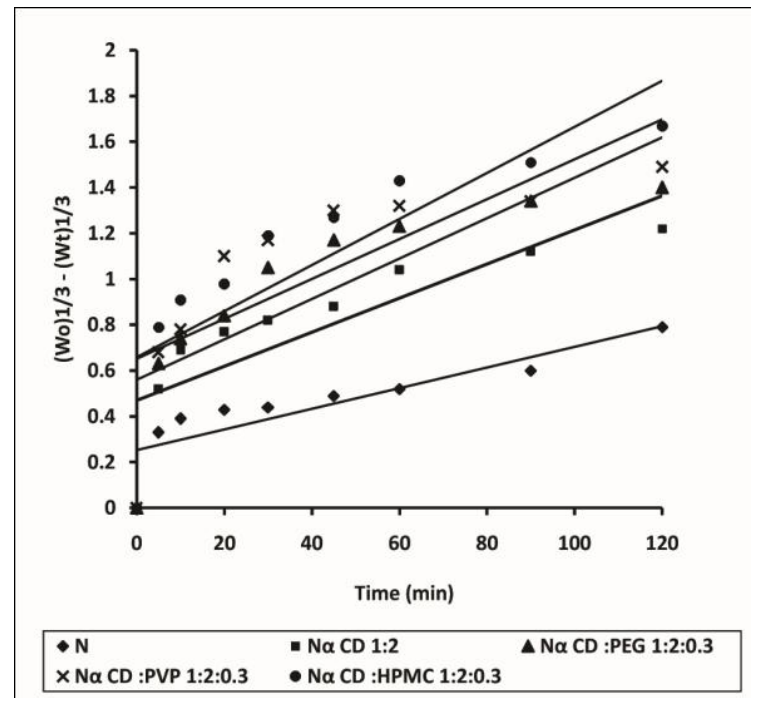

Fig.18. Hixson Crowell Plots of Naproxen and its $\square$-CD complexes Prepared by Physical Mixture Method TABLE 9: Dissolution Parameters of Naproxen and its $\square$ - CD Complexes Prepared By Kneading Method

\begin{tabular}{|c|c|c|c|c|c|c|c|}
\hline $\begin{array}{l}\text { Sl. } \\
\text { No. }\end{array}$ & CD Complex & $\mathbf{D P}_{5 \mathrm{MIN}}$ & $R_{\text {r } 5 \text { min }}$ & $\begin{array}{c}\text { \% Dissolved } \\
\text { in } 10 \mathrm{~min}\end{array}$ & $\mathbf{D E}_{\mathbf{3 0}}$ & $\begin{array}{c}\mathrm{K}_{1} \\
\left(\mathrm{~min}^{-1}\right)\end{array}$ & $\begin{array}{c}\text { Increase } \\
\text { in } K_{1} \\
\text { (No. of } \\
\text { folds) } \\
\end{array}$ \\
\hline 1 & Naproxen & 20.13 & -- & 23.14 & 22.38 & 0.0038 & - \\
\hline 2 & $\mathrm{~N}: \alpha \mathrm{CD} 1: 1$ & 30.32 & 1.48 & 40.63 & 36.34 & 0.025 & 6.61 \\
\hline 3 & $\mathrm{~N}: \alpha \mathrm{CD} 1: 2$ & 53.16 & 2.60 & 62.53 & 58.79 & 0.049 & 13.02 \\
\hline 4 & $\mathrm{~N}: \alpha \mathrm{CD}:$ PEG $1: 1: 0.2$ & 34.56 & 1.69 & 42.13 & 39.15 & 0.028 & 7.27 \\
\hline 5 & $\mathrm{~N}: \alpha \mathrm{CD}:$ PEG 1:2:0.3 & 58.32 & 2.85 & 66.31 & 65.24 & 0.060 & 15.99 \\
\hline 6 & $\mathrm{~N}: \alpha \mathrm{CD}: \mathrm{PVP} 1: 1: 0.2$ & 40.66 & 1.99 & 45.89 & 44.35 & 0.031 & 8.20 \\
\hline 7 & $\mathrm{~N}: \alpha \mathrm{CD}: \mathrm{PVP} 1: 2: 0.3$ & 64.16 & 3.14 & 73.56 & 70.73 & 0.075 & 19.77 \\
\hline 8 & $\mathrm{~N}: \alpha C D: H P M C ~ 1: 1: 0.2$ & 50.32 & 2.46 & 56.83 & 54.12 & 0.042 & 11.15 \\
\hline 9 & $\mathrm{~N}: \alpha \mathrm{CD}: H P M C$ 1:2:0.3 & 70.35 & 3.44 & 79.16 & 75.04 & 0.091 & 23.99 \\
\hline
\end{tabular}

TABLE.10.The Correlation Coefficient $\left(\mathbf{R}^{2}\right)$ Values in the Analysis of Dissolution Data of Naproxen CD Complexes Prepared by Kneading Method as per Zero Order, First Order and Hixson-Crowell Cube Root Models

\begin{tabular}{|c|c|c|c|c|}
\hline \multirow{2}{*}{$\begin{array}{c}\text { Sl } \\
\text { No. }\end{array}$} & \multirow{2}{*}{ Cyclodextrin Complex } & \multicolumn{3}{|c|}{ Correlation Coefficient $\left(\mathbf{R}^{2}\right)$ value } \\
\hline & & Zero Order & First Order & Hixson Crowell \\
\hline 1 & Naproxen & 0.752 & 0.796 & 0.936 \\
\hline 2 & $\mathrm{~N}: \alpha C D 1: 1$ & 0.961 & 0.975 & 0.971 \\
\hline 3 & $\mathrm{~N}: \alpha \mathrm{CD} 1: 2$ & 0.927 & 0.955 & 0.945 \\
\hline 4 & $\mathrm{~N}: \alpha \mathrm{CD}:$ PEG $1: 1: 0.2$ & 0.812 & 0.947 & 0.947 \\
\hline 5 & $\mathrm{~N}: \alpha \mathrm{CD}:$ PEG $1: 2: 0.3$ & 0.807 & 0.878 & 0.932 \\
\hline 6 & $\mathrm{~N}: \alpha \mathrm{CD}: \mathrm{PVP} 1: 1: 0.2$ & 0.794 & 0.926 & 0.923 \\
\hline 7 & $\mathrm{~N}: \alpha \mathrm{CD}: \mathrm{PVP} 1: 2: 0.3$ & 0.799 & 0.952 & 0.942 \\
\hline 8 & $\mathrm{~N}: \alpha C D: H P M C ~ 1: 1: 0.2$ & 0.732 & 0.932 & 0.927 \\
\hline 9 & $\mathrm{~N}: \alpha \mathrm{CD}: H P M C$ 1:2:0.3 & 0.912 & 0.955 & 0.938 \\
\hline
\end{tabular}

TABLE. 11. Dissolution Parameters of Naproxen and its $\square$ - CD Complexes Prepared by Coevaporation Method 
Enhancement of Dissolution rate of Naproxen by Solid

\begin{tabular}{|c|c|c|c|c|c|c|c|}
\hline $\begin{array}{l}\text { Sl. } \\
\text { No. }\end{array}$ & $\begin{array}{l}\text { Cyclodextrin } \\
\text { Complex }\end{array}$ & $\begin{array}{l}\mathrm{DP}_{5} \\
\text { MIN }\end{array}$ & $\begin{array}{l}\mathrm{RD}_{\mathrm{r}} \\
5 \mathrm{~min}\end{array}$ & $\begin{array}{l}\text { \% Dissolved } \\
\text { in } 10 \mathrm{~min}\end{array}$ & $\mathrm{DE}_{30}$ & $\begin{array}{c}\mathrm{K}_{1} \\
\left(\min ^{-1}\right)\end{array}$ & $\begin{array}{c}\text { Increase } \\
\text { in } \mathrm{K}_{1} \\
\text { (No. of } \\
\text { folds) }\end{array}$ \\
\hline 1 & Naproxen & 20.13 & -- & 23.14 & 22.38 & 0.0038 & - \\
\hline 2 & $\mathrm{~N}: \alpha \mathrm{CD} 1: 1$ & 26.23 & 1.28 & 37.36 & 37.33 & 0.050 & 13.33 \\
\hline 3 & $\mathrm{~N}: \alpha \mathrm{CD} 1: 2$ & 44.61 & 2.18 & 52.35 & 40.93 & 0.059 & 15.75 \\
\hline 4 & $\mathrm{~N}: \alpha \mathrm{CD}: \mathrm{PEG} 1: 1: 0.2$ & 30.65 & 1.50 & 39.31 & 44.48 & 0.064 & 16.96 \\
\hline 5 & $\mathrm{~N}: \alpha \mathrm{CD}:$ PEG 1:2:0.3 & 49.32 & 2.41 & 56.31 & 49.77 & 0.071 & 19.83 \\
\hline 6 & $\mathrm{~N}: \alpha C D: P V P 1: 1: 0.2$ & 33.65 & 1.64 & 42.98 & 59.69 & 0.059 & 15.72 \\
\hline 7 & N: $\alpha$ CD:PVP 1:2:0.3 & 53.61 & 2.62 & 62.65 & 62.20 & 0.105 & 27.87 \\
\hline 8 & $\mathrm{~N}: \alpha C D: H P M C ~ 1: 1: 0.2$ & 37.32 & 1.82 & 45.38 & 65.07 & 0.110 & 28.94 \\
\hline 9 & $\mathrm{~N}: \alpha \mathrm{CD}: H P M C$ 1:2:0.3 & 58.23 & 2.85 & 66.61 & 68.66 & 0.128 & 35.82 \\
\hline
\end{tabular}

TABLE. 12.. The Correlation Coefficient $\left(R^{2}\right)$ Values in the Analysis of Dissolution Data of Naproxen CD Complexes prepared by Coevaporation Methods per Zero Order, First Order and Hixson-Crowell Cube Root Models

\begin{tabular}{|c|c|c|c|c|}
\hline \multirow{2}{*}{$\begin{array}{r}\text { Sl } \\
\text { No. }\end{array}$} & \multirow{2}{*}{ Cyclodextrin Complex } & \multicolumn{3}{|c|}{ Correlation Coefficient $\left(\mathrm{R}^{2}\right)$ value } \\
\hline & & Zero Order & First Order & Hixson Crowell \\
\hline 1 & Naproxen & 0.752 & 0.796 & 0.936 \\
\hline 2 & $\mathrm{~N}: \alpha \mathrm{CD} 1: 1$ & 0.863 & 0.967 & 0.975 \\
\hline 3 & $\mathrm{~N}: \alpha \mathrm{CD} 1: 2$ & 0.849 & 0.976 & 0.975 \\
\hline 4 & $\mathrm{~N}: \alpha \mathrm{CD}: \mathrm{PEG} 1: 1: 0.2$ & 0.860 & 0.933 & 0.973 \\
\hline 5 & $\mathrm{~N}: \alpha \mathrm{CD}: \mathrm{PEG} 1: 2: 0.3$ & 0.845 & 0.980 & 0.972 \\
\hline 6 & $\mathrm{~N}: \alpha \mathrm{CD}: \mathrm{PVP} 1: 1: 0.2$ & 0.823 & 0.954 & 0.957 \\
\hline 7 & $\mathrm{~N}: \alpha \mathrm{CD}: \mathrm{PVP} 1: 2: 0.3$ & 0.736 & 0.881 & 0.895 \\
\hline 8 & $\mathrm{~N}: \alpha C D: H P M C$ 1:1:0.2 & 0.913 & 0.992 & 0.985 \\
\hline 9 & $\mathrm{~N}: \alpha \mathrm{CD}: H P M C$ 1:2:0.3 & 0.881 & 0.960 & 0.955 \\
\hline
\end{tabular}

TABLE.13.Dissolution Parameters of Naproxen and its $\square$ - CD Complexes Prepared by Physical Mixture Method

\begin{tabular}{|c|c|c|c|c|c|c|c|}
\hline $\begin{array}{l}\text { S1. } \\
\text { No. }\end{array}$ & Cyclodextrin Complex & $\mathrm{DP}_{5 \mathrm{MIN}}$ & $\begin{array}{l}\mathrm{RD}_{\mathrm{r}} \\
5 \min \end{array}$ & $\begin{array}{l}\text { \% Dissolved } \\
\text { in } 10 \mathrm{~min}\end{array}$ & $\mathrm{DE}_{30}$ & $\begin{array}{c}\mathrm{K}_{1} \\
\left(\min ^{-1}\right)\end{array}$ & $\begin{array}{c}\text { Increase } \\
\text { in } \mathrm{K}_{1} \\
\text { (No. of } \\
\text { folds) }\end{array}$ \\
\hline 1 & Naproxen & 20.13 & -- & 23.14 & 22.38 & 0.0038 & - \\
\hline 2 & $\mathrm{~N}: \alpha \mathrm{CD} 1: 1$ & 22.56 & 1.12 & 30.63 & 23.22 & 0.0186 & 4.92 \\
\hline 3 & $\mathrm{~N}: \alpha \mathrm{CD} 1: 2$ & 30.16 & 1.49 & 38.39 & 36.08 & 0.025 & 6.58 \\
\hline 4 & $\mathrm{~N}: \alpha \mathrm{CD}:$ PEG $1: 1: 0.2$ & 24.86 & 1.23 & 33.13 & 30.67 & 0.0197 & 5.19 \\
\hline 5 & $\mathrm{~N}: \alpha \mathrm{CD}:$ PEG $1: 2: 0.3$ & 35.66 & 1.77 & 40.93 & 40.22 & 0.0213 & 5.62 \\
\hline 6 & $\mathrm{~N}: \alpha \mathrm{CD}: \mathrm{PVP} 1: 1: 0.2$ & 25.56 & 1.26 & 36.88 & 33.32 & 0.023 & 6.199 \\
\hline 7 & $\mathrm{~N}: \alpha \mathrm{CD}: \mathrm{PVP} 1: 2: 0.3$ & 38.16 & 1.89 & 42.56 & 41.47 & 0.022 & 5.83 \\
\hline 8 & $\mathrm{~N}: \alpha C D: H P M C ~ 1: 1: 0.2$ & 28.32 & 1.40 & 40.83 & 34.29 & 0.026 & 6.72 \\
\hline 9 & $\mathrm{~N}: \alpha C D: H P M C$ 1:2:0.3 & 43.32 & 2.15 & 48.38 & 46.25 & 0.024 & 6.26 \\
\hline
\end{tabular}

TABLE .14. The Correlation Coefficient $\left(\mathrm{R}^{2}\right)$ values in the Analysis of Dissolution Data of Naproxen CD Complexes prepared by Physical Mixture Method per Zero Order, First Order and Hixson-Crowell Cube Root Models

\begin{tabular}{|c|l|c|c|c|}
\hline \multirow{2}{*}{$\begin{array}{c}\text { S1 } \\
\text { No. }\end{array}$} & \multirow{2}{*}{ Cyclodextrin Complex } & \multicolumn{2}{|c|}{ Correlation Coefficient } & \multicolumn{2}{c|}{$\left(\mathrm{R}^{2}\right)$ value } \\
\cline { 3 - 5 } & & Zero Order & First Order & Hixson Crowell \\
\hline 1 & Naproxen & $\mathbf{0 . 7 5 2}$ & $\mathbf{0 . 7 9 6}$ & $\mathbf{0 . 9 3 6}$ \\
\hline 2 & N: $\alpha$ CD $1: 1$ & $\mathbf{0 . 8 0 3}$ & $\mathbf{0 . 8 5 9}$ & $\mathbf{0 . 9 6 8}$ \\
\hline 3 & N: $\alpha$ CD $1: 2$ & $\mathbf{0 . 8 3 5}$ & $\mathbf{0 . 8 5 2}$ & $\mathbf{0 . 8 9 6}$ \\
\hline 4 & N: $\alpha$ CD $:$ PEG $1: 1: 0.2$ & $\mathbf{0 . 8 0 5}$ & $\mathbf{0 . 8 3 7}$ & $\mathbf{0 . 9 0 6}$ \\
\hline 5 & N: $\alpha$ CD $:$ PEG $1: 2: 0.3$ & $\mathbf{0 . 8 2 5}$ & $\mathbf{0 . 8 8 6}$ & $\mathbf{0 . 9 0 2}$ \\
\hline
\end{tabular}




\begin{tabular}{|l|l|l|l|l|}
\hline 6 & N: $\alpha$ CD:PVP $1: 1: 0.2$ & $\mathbf{0 . 8 5 2}$ & $\mathbf{0 . 8 7 8}$ & $\mathbf{0 . 8 9 8}$ \\
\hline 7 & N: $\alpha$ CD:PVP $1: 2: 0.3$ & $\mathbf{0 . 8 1 0}$ & $\mathbf{0 . 8 6 6}$ & $\mathbf{0 . 8 9 6}$ \\
\hline 8 & N: $\alpha$ CD:HPMC $1: 1: 0.2$ & $\mathbf{0 . 8 3 9}$ & $\mathbf{0 . 8 9 3}$ & $\mathbf{0 . 9 4 7}$ \\
\hline 9 & N: $\alpha$ CD:HPMC $1: 2: 0.3$ & $\mathbf{0 . 7 7 1}$ & $\mathbf{0 . 8 3 6}$ & $\mathbf{0 . 9 5 2}$ \\
\hline
\end{tabular}

\section{RESULTS AND DISCUSSION}

The dissolution rate of Naproxen $(\mathrm{N})$ from various cyclodextrin solid inclusion complexes was studied in $0.1 \mathrm{~N} \mathrm{HCl}$ and compared with that of un-complexed drug. The dissolution data of $\mathrm{N}-\mathrm{CD}$ complexes are given in Table.1, and the dissolution profiles are shown in Figs.1,4,7,10,13,16,First order plots of the Naproxen $\square-$ CD complexes are shown in Fig. 2,5,8,11,14,17. Hixson-Crowell plots of Naproxen $\square-$ CD complexes are shown in Fig. 3,6,9,12,15,18. The dissolution of Naproxen from the CD complexes was rapid and higher than that of Naproxen as such. The dissolution data were analyzed as per zero-order and first-order kinetics. The dissolution of Naproxen as such and from various cyclodextrin complexes followed first-order kinetics. The ' $r$ ' values were found to be relatively higher in the case of first order model in all the cases (Tables 10,12,14). From the slope of the first order linear plots the dissolution rate constant $\left(\mathrm{K}_{1}\right)$ values were calculated and are given in Tables 9,11,13. The dissolution efficiency $\left(\mathrm{DE}_{30}\right)$ values were calculated. The dissolution parameters of Naproxen and its cyclodextrin complexes are summarized in Tables 9,11,13. All the dissolution parameters (DP 5min, RDr, 5min, \% dissolved in 10 min., $\mathrm{DE}_{30}$ and $\mathrm{K}_{1}$ ) indicated rapid and higher dissolution of Naproxen from the CD complexes when compared to un-complexed drug.

Solid inclusion complexes prepared by kneading method exhibited higher dissolution rate and $\mathrm{DE}_{30}$ values than those prepared by coevaporation in each case. The higher dissolution rates observed with kneaded complexes may be due to better interaction of drug and CD during the kneading process. In each case, the $\mathrm{K}_{1}$ and $\mathrm{DE}_{30}$ values were increased $\mathrm{N}$ : $\alpha \mathrm{CD}$ :HPMC 1:2:0.3 solid dispersion gave a $\mathbf{2 3 . 9 9}$ fold increase in the dissolution rate of Naproxen whereas solid dispersion of Naproxen in alone $\alpha \mathrm{CD}$ (N- $\alpha \mathrm{CD}$ solid dispersion) gave only 6.61 fold increase. Thus combination of Cyclodextrins with water soluble carriers PEG, PVP, HPMC resulted in a greater enhancement in the dissolution rate of Naproxen.

Dissolution of Naproxen from all the solid dispersions followed first order kinetics with correlation coefficient ' $r$ ' above 0.9 (Tables:10,12,14). The increasing order of dissolution rates of solid dispersions of Naproxen are comparable with solid dispersion.

\section{Mechanism of Increased Dissolution Rate of Cyclodextrin Complexes}

The observed increase in the dissolution rate of Naproxen from their cyclodextrin complexes is due to the following possible mechanisms ${ }^{[13,14,15]}$ :

- Due to the possible reduction in particle size and encapsulation of drug into the cyclodextrin cavity.

- The interactions between the hydrophobic part of the guest and the a polar cavity causes dehydration of the hydrophobic guest molecule and its transfer into the cavity, thereby increasing the affinity toward water and hence increasing the dissolution.

- The surfactant like properties of CDs can also be postulated to explain the higher dissolution rate of the complexes.

- CDs can also reduce the interfacial tension between the solid particles of drug and the dissolution medium, leading to a greater rate of dissolution.

\section{CONCLUSION}

The dissolution rate and dissolution efficiency of Naproxen could be enhanced several times by their solid dispersion in cyclodextrin alone and in combination with hydrophilic polymers such as PEG, PVP, HPMC. Cyclodextrin particularly HPMC was found to be good carrier giving solid dispersions with enhanced dissolution rate and efficiency, several times higher than those of pure drug ${ }^{[14,15,16]}$.. Thus, solid dispersion in Cyclodextrin is recommended as an effective and efficient technique for enhancing the dissolution rate, dissolution efficiency of Naproxen. Cyclodextrin are inert, safe and non-toxic excipients that are currently used in compressed tablet formulations. These can be used as efficient carriers in solid dispersion techniques to enhance the dissolution rate of insoluble and poorly soluble drugs.

\section{REFERENCES}


[1] Chawla A, Sharma P, PawarP,Eudragit S-100 coated sodium alginate microspheres of naproxen sodium: Formulation, optimization and in vitro evaluation,Acta Pharm. 2012 Dec 1;62(4):529-45

[2] Zhu Q, Toth SJ, Simpson GJ, Hsu HY, Taylor LS,Crystallization and dissolution behavior of naproxen/polyethylene glycol solid dispersions.Journal of Physical Chemistry $\quad$ B. 2013 Feb 7;117(5):1494-500.

[3] Guo Z, Liu XM, Ma L, Li J, Zhang H, Effects of particle morphology, pore size and surface coating of mesoporous silica on Naproxen dissolution rateenhancement.ColloidsSurfaceBiointerfaces. 2013 Jan $1 ; 101: 228-35$.

[4] Hsu HY, Toth SJ, Simpson GJ, Taylor LS, Harris MT, Effect of substrates on naproxenpolyvinylpyrrolidone solid dispersions formed via the drop printing technique..JournalofPharmaceutical Sciences. 2013 Feb;102(2):638-48.

[5] Raijada D, Bond AD, Larsen FH, Cornett C, Qu H,Exploring the solid-form landscape of pharmaceutical hydrates: transformation pathways of the sodium naproxenanhydrate-hydrate system. Journal of Pharma Research. 2013 Jan;30(1):280-9.

[6] Kayaert P, Van den MooterG. An investigation of the adsorption of hydroxypropylmethyl cellulose 29105 $\mathrm{mPa} \quad \mathrm{s}$ and polyvinylpyrrolidone K90 around Naproxen nanocrystals."Journal of Pharmaceutical Sciences. 2012 Oct;101(10):3916-23

[7] Paudel A, Nies E, Van den MooterG. Relating hydrogen-bonding interactions with the phase behavior of naproxen/PVP K 25 solid dispersions: evaluation of solution-cast and quench-cooled films.Molecular Pharmacy. 2012 Nov 5;9(11):3301-17

[8] Ameli A, Alizadeh Nanostructured conducting molecularly imprinted polymer for selective uptake/release of naproxen by the electrochemically controlled sorbent.AnalyticalBiochemistry. 2012 Sep 15;428(2):99-106

[9] Kayaert P, Van den MooterG.Is the amorphous fraction of a dried nanosuspension caused by milling or by drying? A case study with Naproxen.EurJournal of Pharm Biopharmaceutics. 2012 Aug; 81(3):650-6.

[10] Ando S, Kikuchi J, Fujimura Y, Ida Y, Higashi K,Physicochemical characterization and structural evaluation of a specific 2:1 cocrystal of naproxen-nicotinamide.Journal of Pharmaceutical Sciences. 2012 Sep;101(9):3214-21.

[11] Mati SS, Mondal TK, Dhar S, Chall S, Differential contribution of Igepal and CnTAB micelles on the photophysics of nonsteroidal drug Naproxen ,SpectrochimActa A Mol Biomol Spectrosc. 2012 Jun $15 ; 92: 122-30$

[12] Čalija B, Milić J, Cekić N, Krajišnik D, Chitosan oligosaccharide as prospective cross-linking agent for naproxen-loaded Ca-alginate microparticles with improved $\mathrm{pH}$ sensitivity.Drug Development in Industrial Pharmacy. 2013 Jan;39(1):77-88.

[13] Ayenew Z, Paudel A, Van den MooterG. Can compression induce demixing in amorphous solid dispersions? A case study of naproxen-PVP K25EurJournal of Pharm Biopharmceutics. 2012 May;81(1):207-13.

[14] Tian Q, Ren F, Xu Z, Xie Y, Zhang S. Preparation of high solubilizablemicroemulsion of naproxen and its solubilization mechanism International Journal of Pharmacy. 2012 Apr 15;426(1-2):202-10.

[15] Rao KR, Nagabhushanam MV, Chowdary KP, In vitro Dissolution studies on Solid Dispersions of Mefenamic Acid, Indian J Pharm Sci. 2011 Mar; 73(2):243-7

[16] .Lachman L. In the Theory and practive of Industrial Pharmacy.Lea and Febiger, Philadelphia.1976, p 101. 


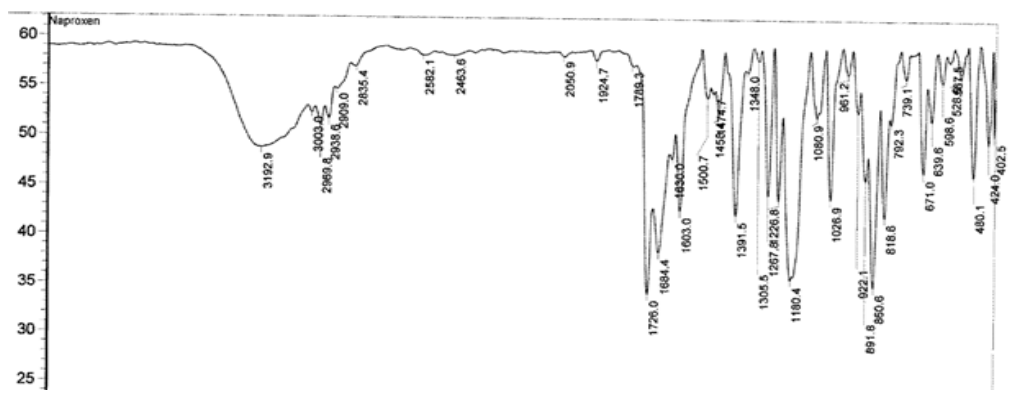

(i)

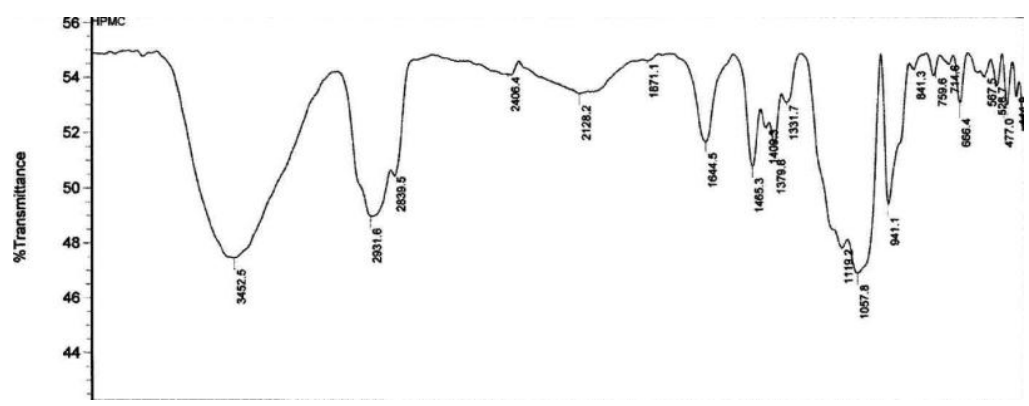

(ii)

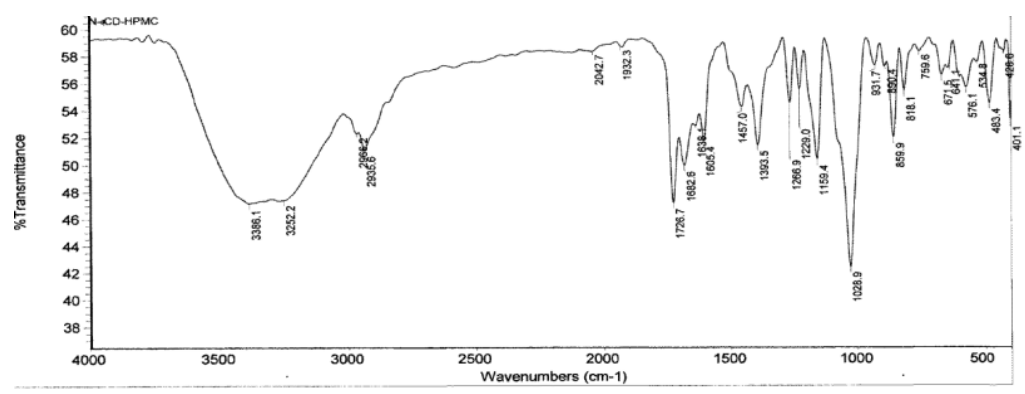

(iii)

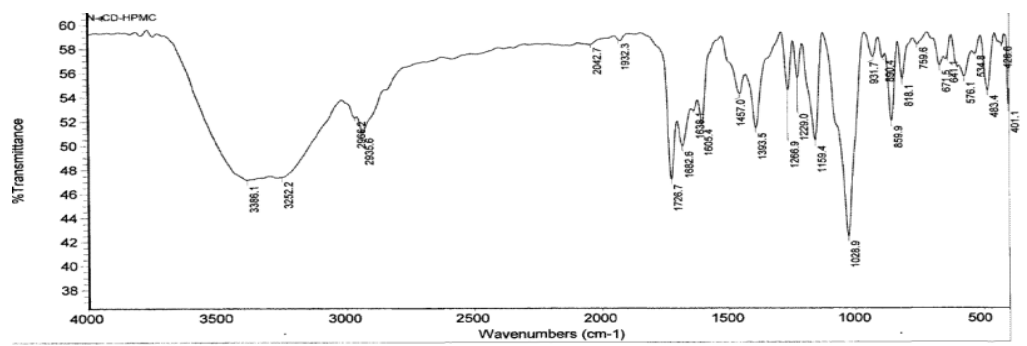

(iv)

Fig : 19. IR Spectra of (i) Naproxen (ii) $\alpha$-CD (iii) HPMC (iv) Naproxen : $\alpha-C D$ HPMC 


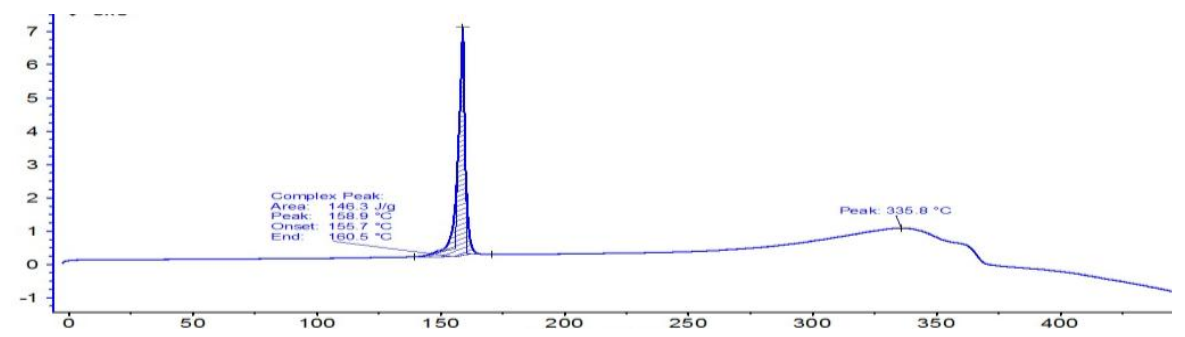

(i)

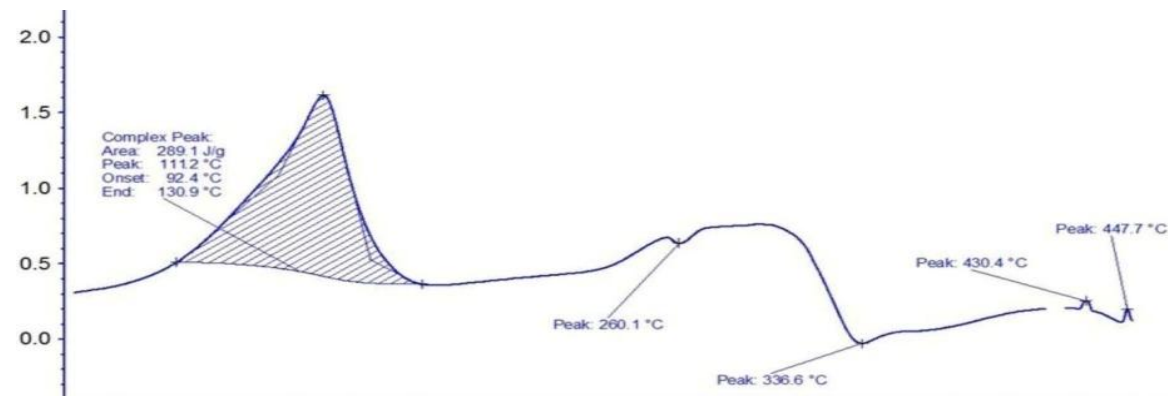

(ii)

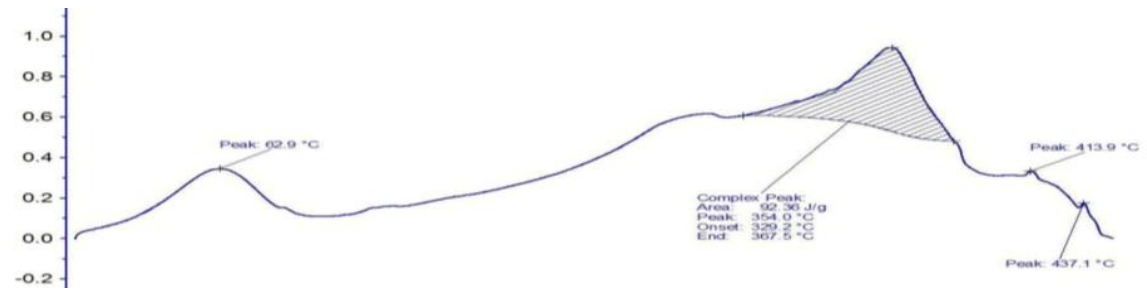

(iii)

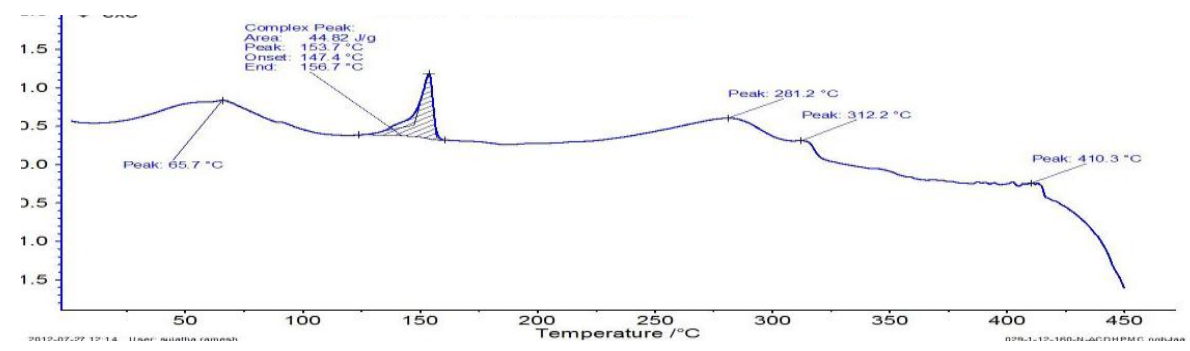

(iv)

Fig : 20. DSC Spectra of (i) Naproxen (ii) $\alpha-C D$ (iii) HPMC iv) Naproxen: $\alpha C D-H P M C$ 


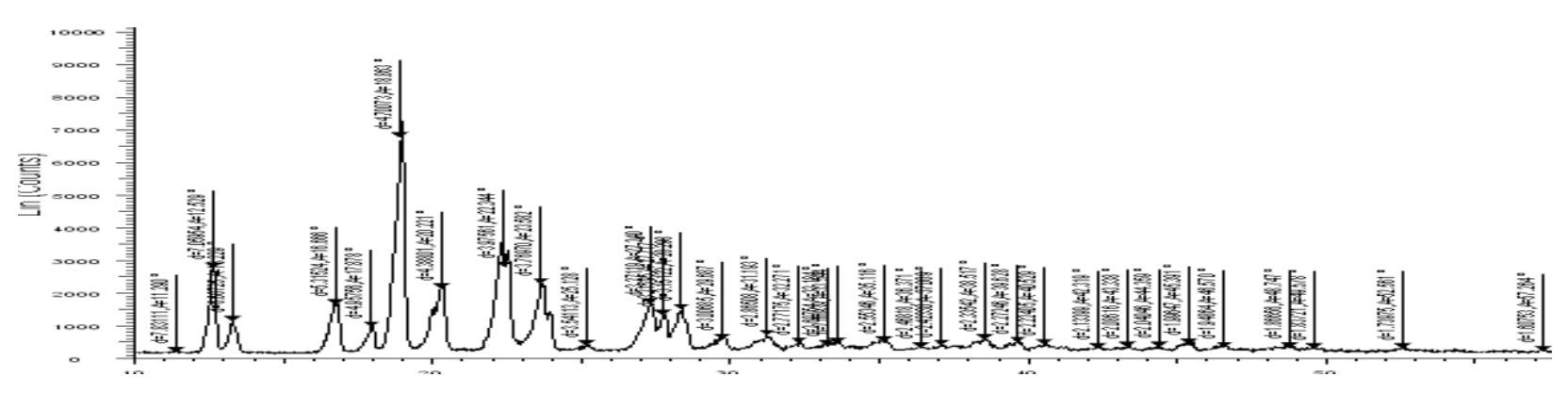

(i)

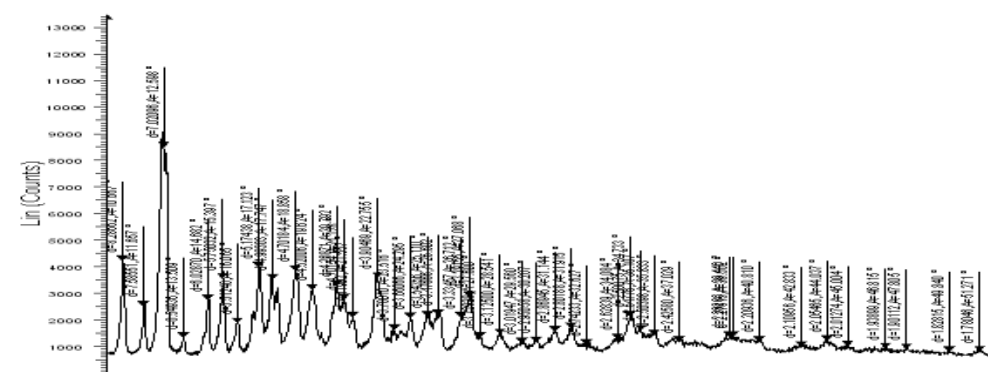

(ii)

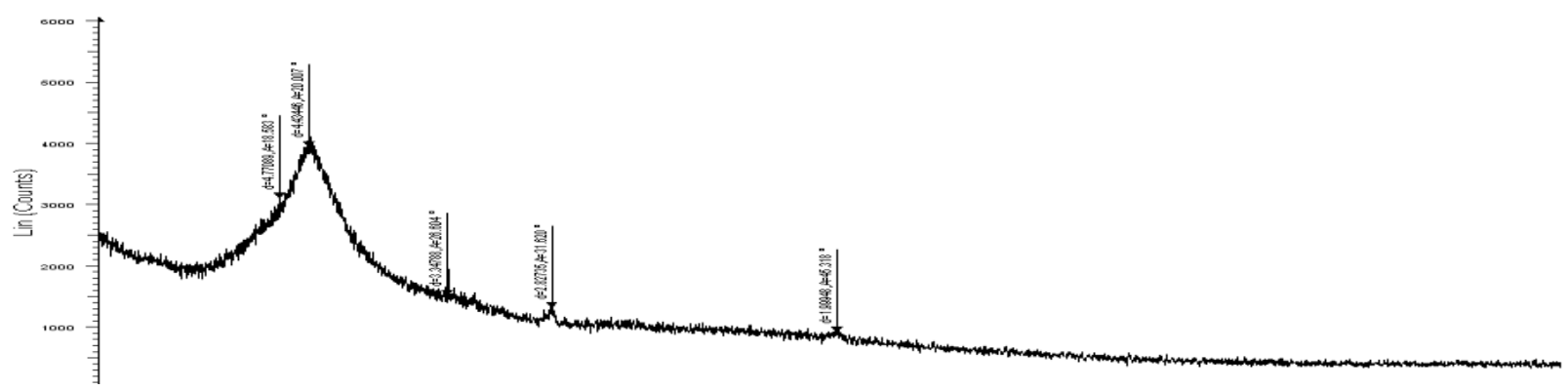

(iii)

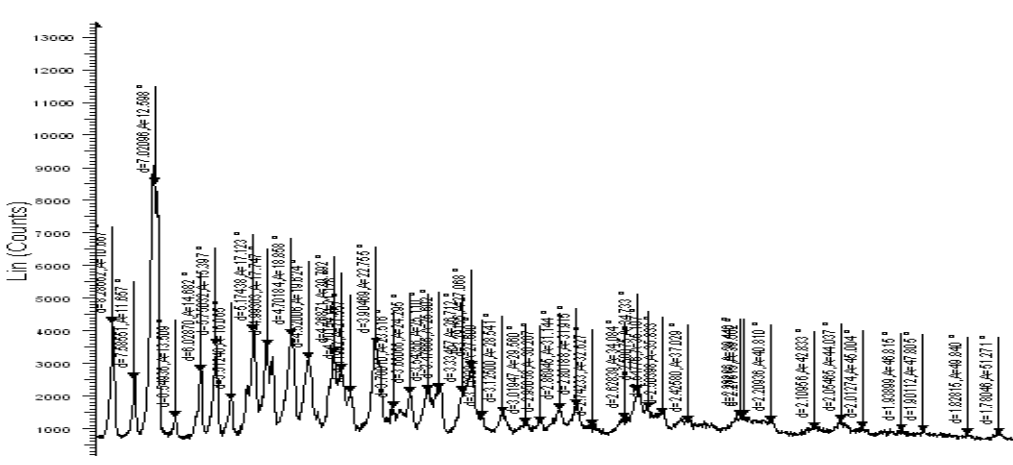

(iv)

Fig :21. XRD Spectra of (i)Naproxen (ii) $\alpha-C D \quad$ (iii) HPMC iv) Naproxen : $\alpha C D-H P M C$ 


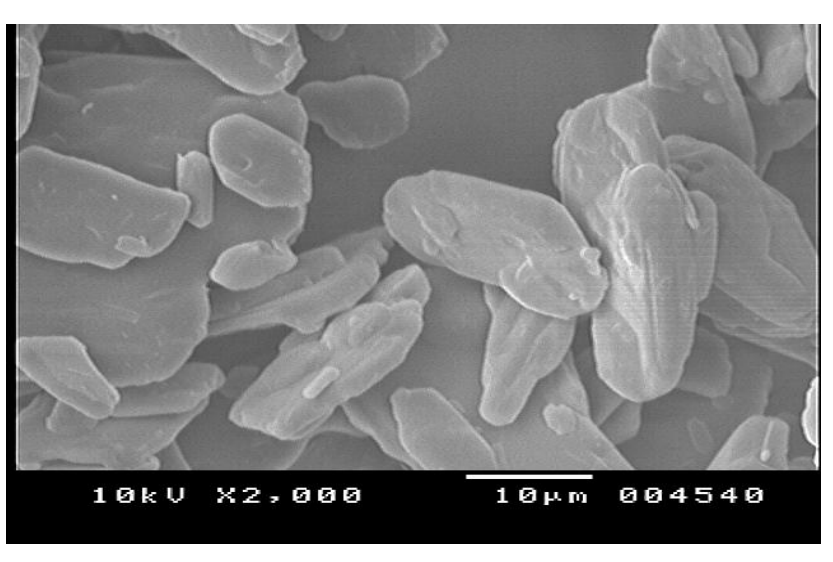

(I)

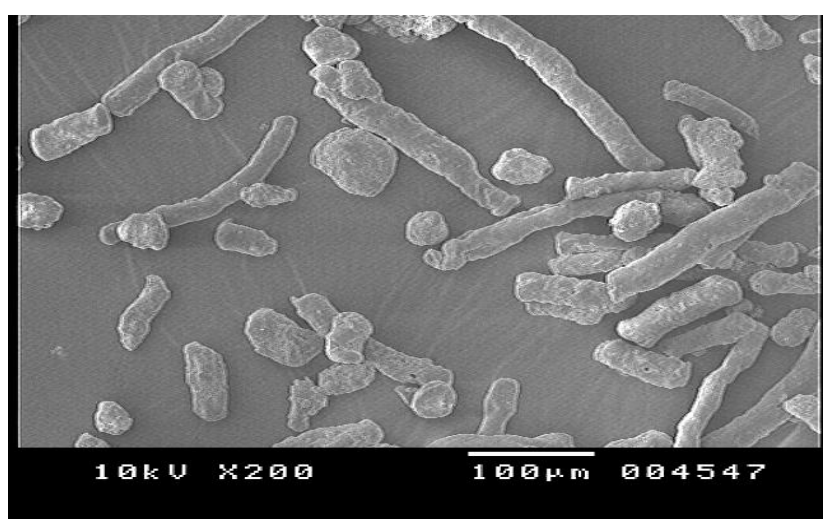

(III)

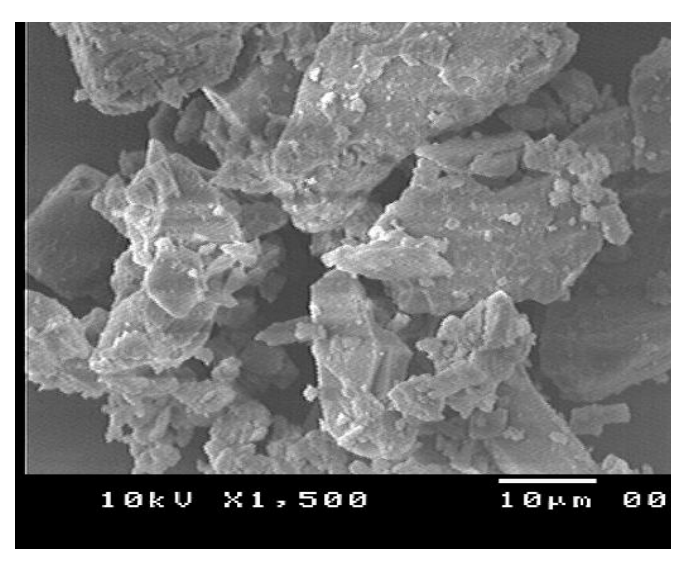

(II)

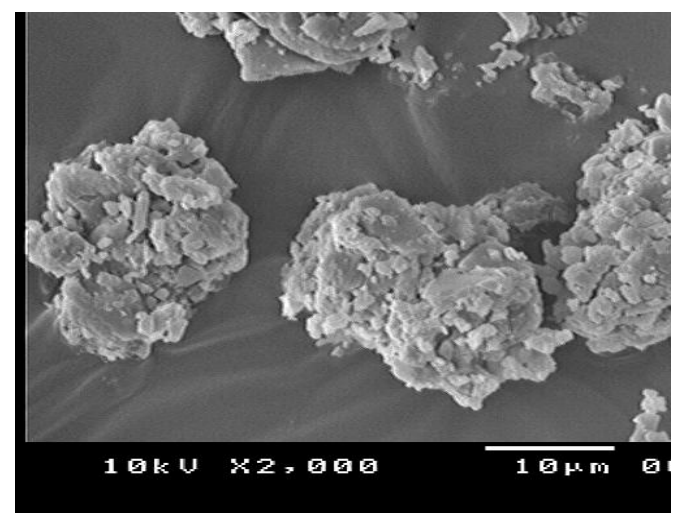

(IV)

Fig : 22.SEM images (i)Naproxen (ii) $\alpha$-CD (iii) HPMC iv) Naproxen : $\alpha C D-$ HPMC 